\title{
Energy Barrier Reduction for the Double Proton-Transfer Reaction in Guanine-Cytosine DNA Base Pair on a Gold Surface
}

R. R. Q. Freitas, R. Rivelino, F. de B. Mota, Gueorgui Kostov Gueorguiev and C. M. C. de Castilho

\section{Linköping University Post Print}

\section{Tweet}

N.B.: When citing this work, cite the original article.

Original Publication:

R. R. Q. Freitas, R. Rivelino, F. de B. Mota, Gueorgui Kostov Gueorguiev and C. M. C. de Castilho, Energy Barrier Reduction for the Double Proton-Transfer Reaction in GuanineCytosine DNA Base Pair on a Gold Surface, 2015, The Journal of Physical Chemistry C, (119), 27, 15735-15741.

http://dx.doi.org/10.1021/acs.jpcc.5b04149

Copyright: American Chemical Society http://pubs.acs.org/

Postprint available at: Linköping University Electronic Press http://urn.kb.se/resolve?urn=urn:nbn:se:liu:diva-120452 
http://pubs.acs.org/doi/abs/10.1021/acs.jpcc.5b04149

Energy Barrier Reduction for the Double Proton-Transfer Reaction in GuanineCytosine DNA Base Pair on a Gold Surface

R. R. Q. Freitas,${ }^{\dagger}$ R. Rivelino, ${ }^{* \dagger}$ F. de B. Mota,${ }^{\dagger}$ G. K. Gueorguiev ${ }^{\ddagger}$ and C. M. C. de Castilho*广§

† Instituto de Física, Universidade Federal da Bahia, 40170-115 Salvador, Bahia, Brazil

$\$$ Department of Physics, Chemistry and Biology, IFM, Linköping University, 58183

Linköping, Sweden

§ Instituto Nacional de Ciência e Tecnologia em Energia e Ambiente - INCT\&EA, Campus Universitário da Federação, Universidade Federal da Bahia, 40170-280

Salvador, Bahia, Brazil 
ABSTRACT. We investigate, by means of first-principles calculations, the impact of a gold surface on the proton-transfer of the guanine-cytosine (GC) DNA base pair. Our calculations employ density functional improvements to correct van der Waals interactions and properly treat a weakly bound GC pair at an $\mathrm{Au}(111)$ surface. We adopted the simultaneous double proton-transfer (SDPT) mechanism proposed by Löwdin, which may lead to a spontaneous mutation in the structure of DNA from specific tautomerization involving the base pairs. Our calculated differences in the energetics and kinetics of the SDPT in the GC pair, when in contact with an inert gold surface, indicate a reduction of about $31 \%$ in the activation energy barrier of the $\mathrm{GC} / \mathrm{Au}(111)$ tautomeric equilibrium. This finding gives strong evidence that tautomerism of DNA base pairs, binding to a noble surface, may be indeed relevant for the assessment of a possible point mutation, which could be induced by the presence of gold nanoparticles during DNA replication.

Keywords: nucleobase tautomerization; nanotoxicology; van der Waals interactions; density functional theory; double proton-transfer; gold surface; mutation 


\section{INTRODUCTION}

The adsorption of organic molecules onto the surface of abiogenic minerals was probably one of the underlying processes behind the origin of life $e^{1,2}$ on Earth around 3.8 billion years ago. ${ }^{3}$ Now, as it is well known from heterogeneous catalysis, ${ }^{4}$ molecular adsorption is the fundamental step towards obtaining desired products from appropriate reactants. In general, the adsorption mechanism is primarily dominated by van der Waals (vdW) interactions between an adsorbed molecule and the surface atoms, which induce small changes in the electronic structure of the adsorbate. ${ }^{5}$ Otherwise, the electronic structure of the adsorbed molecule can be strongly affected thus originating chemical bonds with the surface. ${ }^{4}$ These processes are of paramount importance in biophysical chemistry, since they represent, together with the electromagnetic radiation, most of the interactions of a biological system under environmental conditions. For these reasons, understanding how molecules of biological interest can behave, when in contact with surface atoms or nanoparticles, is an issue of increasing interest in biological sciences. ${ }^{6-8}$

First-principles computational simulations ${ }^{9-15}$ may aid to unravel the underlying causes of intricate biological phenomena occurring at the atomic level. Moreover, owing to the major advances in atomic force microscopy (AFM), ${ }^{5}$ as well as scanning tunneling microscopy (STM), ${ }^{16}$ the interaction of biological molecules with a metal surface $^{17}$ has been a hot topic in metal/organic interfaces at the single-molecule level. Because of both theoretical and experimental advances at the molecular level, the interest in self-assembly of organic molecules at metal and oxide surfaces has also been

relighted in nanoscience. ${ }^{18-21}$ Recently, a molecular description of $(S)$-glutamic acid self-assembly adsorbed on $\operatorname{Ag}(100)$ at low-temperature ${ }^{22}$ has been made possible via STM imaging and first principles calculations. The particular problem of this molecule 
on $\operatorname{Ag}(100)$ is of great interest since it is adsorbed in its neutral form, leading to supramolecular structures from hydrogen bonding, which is also of biological relevance.

Another appealing case in this field has been the preparation of supramolecular systems on metallic surfaces. ${ }^{16}$ This is also the case of chiral pentamers from all-transretinoic acid on the $\mathrm{Au}(111)$ surface $^{18}$ at room temperature. These structures are clearly stabilized by hydrogen bonding, although they are only favored on $\mathrm{Au}(111)$ owing to the inertness and favorable crystallographic direction of this surface. Indeed, the effects of a noble substrate, such as $\mathrm{Au}(111)$, have been investigated for a variety of organic molecules in the past decade by scanning probe techniques. ${ }^{16,19-21}$ As it has been noticed, a noble metal surface is a worthy substrate for self-assembled molecular layering, mainly because of their mild chemical reactivity and high atomic surface mobility. Furthermore, its high surface electron density represents a charge reservoir, which may inhibit electrochemical processes. ${ }^{23}$

More related to the main aim of the present paper, the formation of planar hydrogen-bonded networks of DNA nucleobases, such as guanine $(\mathrm{G})^{16}$ and cytosine (C) ${ }^{19}$ adsorbed on an $\mathrm{Au}(111)$ surface, has been an issue of great interest. As is noticed in these early works, the symmetry of the functional groups of the $\mathrm{C}$ and $\mathrm{G}$ molecules allows the self-assembly of ordered network, although in the case of the $\mathrm{C}$ molecules their supramolecular structures miss long-range order. ${ }^{19}$ This finding may be also related to the differential adsorption of nucleic acid bases. Sowerby et al. ${ }^{2}$ have experimentally determined the equilibrium adsorption isotherms of the nucleobases on the surface of crystalline graphite, for which the $\mathrm{G}$ molecule exhibits a much stronger adsorption than C. Other important point to be considered here about the nucleobases is that they undergo tautomerization. ${ }^{24}$ Kong et al. ${ }^{25}$ have demonstrated, via STM imaging 
and density-functional calculations, that the tautomeric equilibrium of $\mathrm{G}(\mathrm{G} / 9 \mathrm{H}$ $\mathrm{G} / 7 \mathrm{H})$ is facilitated on $\mathrm{Au}(111)$ by heating. Therefore, the mismatch of base pairing may be induced in the presence of gold. Even though the adsorption of the separated nucleobases on solid surfaces appears to have been investigated in detail, their base pairing is not yet fully understood in a detailed level.

In this paper, we investigate the role of an $\mathrm{Au}(111)$ surface in the proton-transfer (PT) reactions of the Watson-Crick GC base pair by means of first principles calculations. ${ }^{26-30}$ We adopt the mechanism, firstly proposed by Löwdin, ${ }^{31}$ in which the transformation of $\mathrm{G}$ and $\mathrm{C}$ into their most common tautomers ${ }^{24} \mathrm{G}^{*}$ and $\mathrm{C}^{*}$ may lead to a spontaneous mutation ${ }^{32}$ in the structure of DNA. We have chosen this mechanism because, quite recently, Vecchio et al. ${ }^{33}$ have pointed out mutagenic effects of gold nanoparticles in the phenotype of Drosophila melanogaster. Moreover, our computational study is boosted by recent experiments conducted by $\mathrm{Li}$ et al., ${ }^{34}$ which correlate mutagenicity and tautomerism in the anti-HIV nucleoside KP1212. Therefore, after obtaining the initial and final states of the GC base pair, we determine the minimum energy paths (MEPs) and transition state (TS) ${ }^{35-37}$ for the tautomeric equilibrium of $\mathrm{GC}$ in the gas phase and at $\mathrm{Au}(111)$. The van der Waals interactions between the base pair and the surface, as well as for describing the GC hydrogen bonding, are properly taken into account in our calculations. ${ }^{30} \mathrm{We}$ demonstrate that tautomeric equilibrium barrier is significantly lowered in the presence of the $\mathrm{Au}(111)$ surface. This finding implies a close relationship with the mismatch of GC in DNA, when in contact with a noble metal surface.

\section{THEORETICAL METHODS}


Electronic Structure and Geometry. Our calculations were based on recent density functional theory improvements, ${ }^{30}$ which properly take into account both van der Waals interactions and lattice constants of solids, as implemented in the VASP code. ${ }^{26-28}$ The electron-ion interactions were described using the projected augmented wave (PAW) potentials. ${ }^{28}$ Plane-wave cut off energies of $700 \mathrm{eV}$ and self-consistency smaller than $10^{-5} \mathrm{eV}$ were adopted in the electronic structure calculations. We have obtained the structural relaxation of a GC pair on an $\mathrm{Au}(111)$ surface as well as the corresponding separate moieties. The gold surface was modeled by a 162-atom slab divided into six layers, each one containing 27 atoms and a vacuum layer with $16.0 \AA$ width. During the relaxation process, the three top layers were allowed to relax, keeping the three subsequent layers fixed, occupying the bulk positions. The Brillouin zone was sampled using a $3 \times 3 \times 1$ Monkhorst-Pack set for the $\mathrm{Au}(111)$ surface, GC/Au(111), and only the $\Gamma$ point for the isolated GC pair. At the first stage of the relaxation procedure, the geometries were optimized using the $\mathrm{PBE}^{29}$ exchange and correlation functional until the forces became smaller than $0.05 \mathrm{eV} / \mathrm{A}$. Hence, the optimized geometries were taken as starting point for obtaining the new equilibrium positions including dispersion interactions, using the optB86b vdW-density functional ${ }^{30}$. At this second stage of these calculations, all gold atoms were kept fixed while the GC pair was fully allowed to relax.

Adsorption and Charge Transfer. The binding energy was calculated from the usual definition:

$$
E_{b}=-\left(E_{G C / A u(111)}-E_{A u(111)}-E_{G C}\right)
$$

where the rhs first term is the total energy of the GC/Au(111) system, the second term is the total of the clean gold surface, and the last one corresponds to the total energy of the isolated GC pair. According to this definition, a positive binding energy value indicates 
an energetically favorable adsorbate. We have also analyzed the electronic structure at the metal/molecule interface by evaluating the charge density difference as well as the electrostatic potential. The charge difference was calculated as follows:

$$
\Delta \rho=\rho_{G C / A u(111)}-\rho_{A u(111)}-\rho_{G C}
$$

where the rhs first term is the charge density of the $\mathrm{GC} / \mathrm{Au}(111)$ system, while the second and third term, respectively, represent the charge density of the clean $\mathrm{Au}(111)$ slab and isolated GC pair. Additionally, it was calculated the charge transfer between the GC pair and the $\mathrm{Au}(111)$ slab by means of the Bader analysis on the charge density grid. ${ }^{39}$

Minimum Energy Paths and Transition State Calculation. The simultaneous double proton transfer (SDPT) in the GC pair, isolated and adsorbed on an Au(111) surface, was investigated through the nudged elastic band (NEB) algorithm. ${ }^{35,36}$ The adiabatic minimum energy paths (MEPs) from a sequence of discrete images was produced. Each image corresponds to a specific geometry of the GC atoms along their pathway from the fixed initial state $(\mathrm{GC})$ to the fixed final state $\left(\mathrm{G}^{*} \mathrm{C}^{*}\right)$. The maximum along this path is a first-order saddle point in the potential energy surface. The standard NEB calculations were performed for approximately 100 ionic steps to achieve an approximate convergence for a specific MEP. In order to obtain a rigorous convergence for the transition state (TS) associated to this specific MEP, the calculations were followed by applying the climbing-image NEB algorithm (CI-NEB). ${ }^{37}$ In this direction, we have obtained the kinetics of the SDPT of GC and GC/Au(111).

\section{RESULTS AND DISCUSSION}


First-principles Modeling of the GC Base Pair at Au(111). Although the present work is focused on the SDPT in the GC base pair adsorbed on an $\mathrm{Au}(111)$ surface, we first investigate some structural, energetic and electronic properties of the base pairing for completeness (a benchmark is reported in the Supplementary Information (SI)). In Figure 1, we display the most stable configuration (on the hcp site) obtained for the adsorbed GC pair on $\mathrm{Au}(111)$, taking into account vdW-density functional improvements ${ }^{30}$ during the relaxation procedure. Our gold surface is modeled by a six-layer slab each containing 27 atoms. In Table S1, we list the calculated average distance between the GC pair and the gold surface, binding energy and charge transfer (see definitions of these properties in the SI), calculated for the distinct sites (bridge, hcp, fcc, and top). We note that the major differences by including vdW interactions appear in the calculated binding energy as well as in charge transfer. While the average distance between GC and $\mathrm{Au}(111)$ decreases by ca. 9.7\%, in the case of the hcp site, the binding energy increases almost eight times and the charge transfer per heavy atoms increases around 36\%. Our values are in line with recent theoretical results for a single G molecule on $\mathrm{Au}(111){ }^{38}$

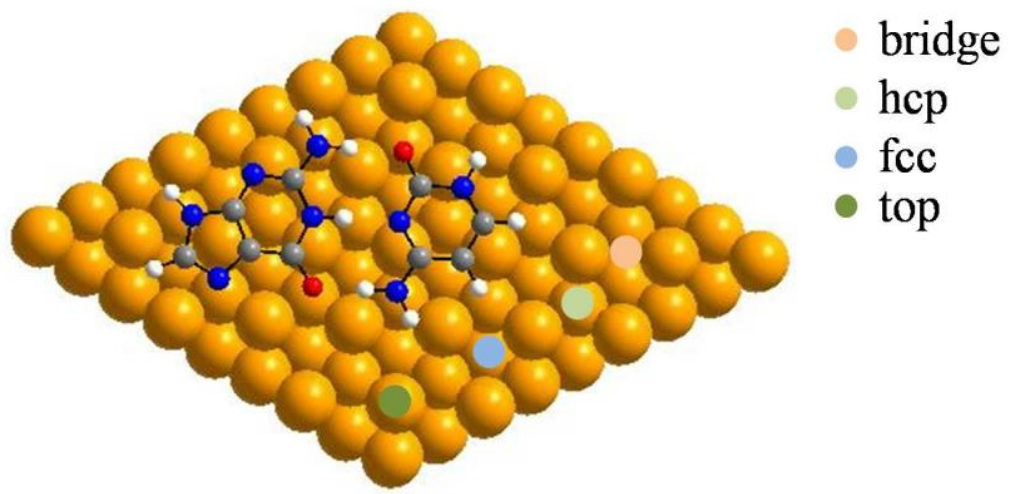

Figure 1. Optimized GC base pair on the hcp site (the strongest calculated binding energy with vdW-DF corrections ${ }^{30}$ ) of an $\mathrm{Au}(111)$ surface. The molecules can be also 
adsorbed with their hexagonal rings in four distinct possibilities: bridge, fcc, and top sites (see SI).

Dispersion interactions between the GC pair with gold surface indeed lead to appreciable variations in the calculated charge density difference, as well as in the equilibrium geometry of the base pair. The $x y$-plane averaged charge redistributed in $\mathrm{GC} / \mathrm{Au}(111)$ interface is plotted in Figure 2, as a function of distance along the $z$ direction interface unit cell. In Figure 2a (without dispersion interactions), we notice that the charge density difference exhibits only very small fluctuations from bottom, at the fifth layer of the gold slab, to a little stronger depletion on the sixth layer, followed by an increase in the GC pair region (between -0.1 and 0.1 e/ $\AA$ ). Our computed charge transfer per heavy atoms between GC and metal surface, using the Bader analysis, ${ }^{39}$ is of $0.2 \times 10^{-2} e$ in this case (without considering wdW interactions ${ }^{29}$ ). Also, an increase in $\Delta \rho$ is observed in the hydrogen bonding region between the GC pair. Most importantly, the impact of the dispersion effects in the calculations (considering vdW interactions ${ }^{30}$ ) implies significant changes in the charge density difference, mainly at the interface region between $\mathrm{GC}$ and the $\mathrm{Au}(111)$ surface, as displayed in Figure 2b. A stronger depletion occurs at this interface, as well as a significant amount of charge density is interchanged between the GC pair. Now, a depletion in $\Delta \rho$ is also observed around the hydrogen bonding region. In this case, the computed charge transfer per heavy atoms between GC and gold surface is of $0.3 \times 10^{-2} e$. In general, the increase in the charge density is essentially more spread on the $\mathrm{Au}(111)$ surface (between -0.2 and $0.2 \mathrm{e} / \AA$ ) and the PT region exhibits a localized charge density depletion (cyan iso-surface). These findings indicate that a gold surface may dramatically affect the PT mechanism in the GC pairs. 

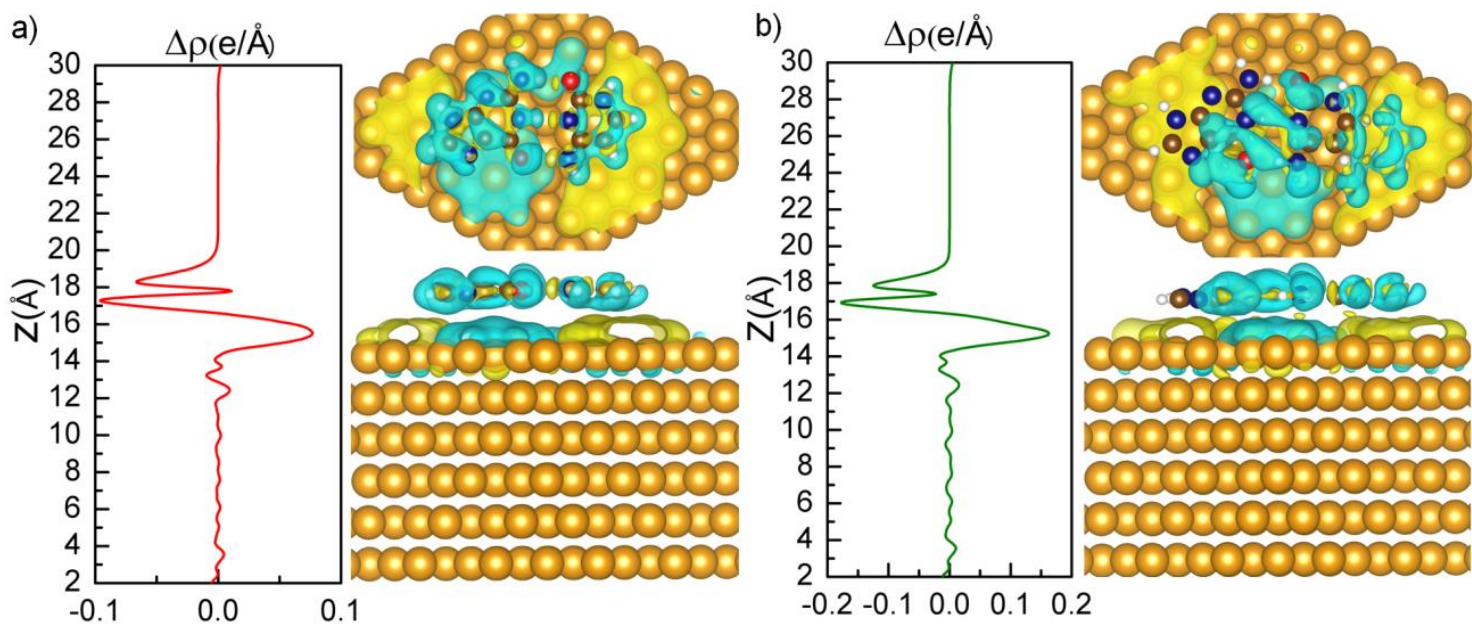

Figure 2. Calculated charge density difference for the GC pair adsorbed on hcp site of the $\mathrm{Au}(111)$ surface using density-functional methods: (a) without dispersion interactions ${ }^{29}$ and (b) with dispersion interactions. ${ }^{30}$ Plot on the left is the $x y$-plane averaged charge difference, with charge difference $(\Delta \rho)$ on the $x$-axis and distance in $z$ direction of the interface unit cell on $y$-axis. Plot on the right represents the threedimensional iso-surface of charge density difference. Yellow iso-surface indicates an increase and cyan iso-surface indicates a depletion in the charge density redistribution.

In addition to the charge density difference displayed in Figure 2, we analyze the electronic structure at the metal/GC interface by evaluating the electrostatic potential from vdW-DF calculations. The charge density and the electrostatic potential are averaged in planes parallel to the $\mathrm{Au}(111)$ slab, leading to the one-dimensional potential curve displayed in Figure 3. As noticed before, the alternating regions of charge accumulation $(\Delta \rho>0)$ and charge depletion $(\Delta \rho<0)$ appear only near the GC/Au(111) interface from the fifth layer to the GC pair. Interestingly, the GC adsorption leads to a small reduction in the metal work function, when compared to other metal/molecule interfaces. ${ }^{40}$ In Table S2, we report the calculated metal work function for the molecular adsorption at different sites of the $\mathrm{Au}(111)$ surface and different levels of approximation. As a result of this apparently weak adsorption, the DFT estimate of the 
GC band gap upon adsorption indicates only a very small reduction in comparison to the value of $2.40 \mathrm{eV}$ for the isolated system.

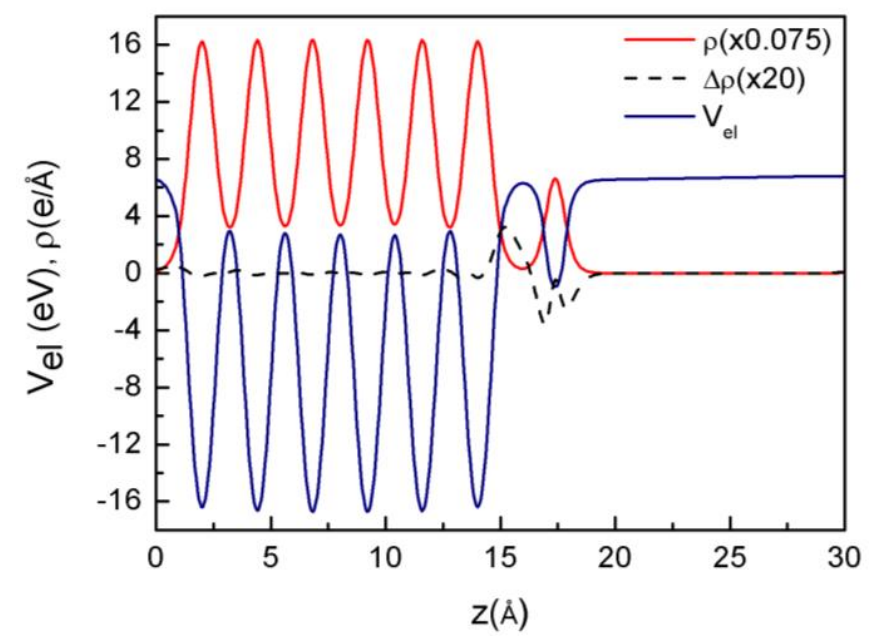

Figure 3. One-dimensional electrostatic potential, total electron density, and charge density difference across the gold slab, calculated using vdW-DF. ${ }^{30}$ The total electron density is multiplied by a factor 0.075 and $\Delta \rho$ by a factor of 20 , for a better visualization.

In Figure 4, we show the partial density of electronic states of the adsorbed GC pair on the $\mathrm{Au}(111)$ surface. Although there exists an energy gap corresponding to the GC PDOS (green line), we notice a small increase in the total DOS, referring to the GC/Au(111) system, as compared to the PDOS of gold (red line). In fact, this electronic contribution to the total DOS is related to the charge transfer from the GC pair to the gold surface. Also, the highest occupied molecular orbital (HOMO) of both the isolated and adsorbed GC pair are displayed in the right panel of Figure 4. As can be seen, the HOMO charge density of the adsorbed pair is qualitatively different from that of the isolated pair. While in the case of the isolated GC pair it is concentrated in the G molecule, in the case of the adsorbed GC pair HOMO charge density is spread on both 
molecules. Again, these results may indicate that the Au(111) surface is not too inert as should be expected, and directly impacts on the tautomerism of GC.

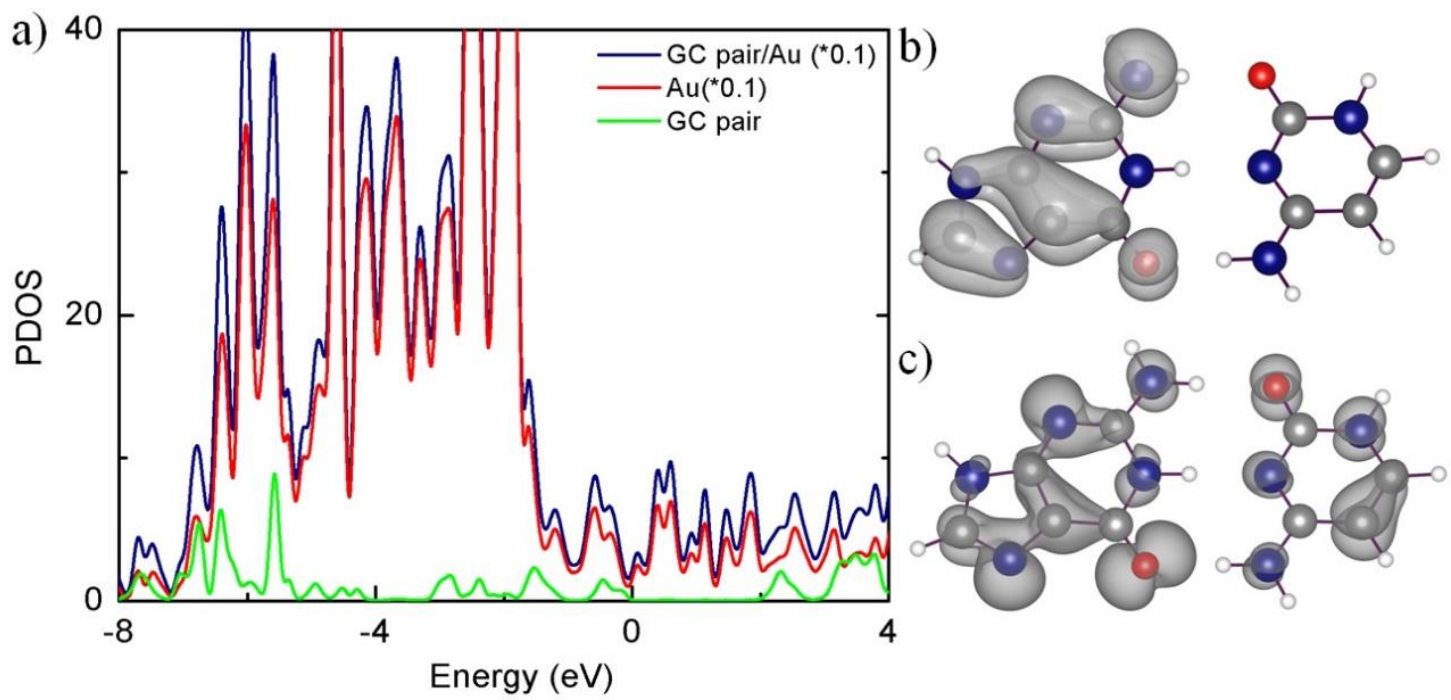

Figure 4. (a) Partial density of electronic states (PDOS) for the GC pair adsorbed at the $\mathrm{Au}(111)$ surface (hcp site), calculated with vdW-DF. ${ }^{30}$ The Fermi level is set at the origin of the energy scale. The contribution of the surface was reduced 10 times for visual convenience. (b) and (c) HOMO charge densities of the isolated and adsorbed GC pair, respectively.

Impact of Proton-Transfer Reactions in DNA Base Pairs. The proton tunneling in DNA base pairs has long been recognized as the ultimate atomic process behind the spontaneous mutations occurring after DNA replications. ${ }^{31}$ Recently, random mutations arising during DNA replication in normal, noncancerous stem cells have been associated to the majority of cancer risk. ${ }^{32}$ During the replication, the protons involved in the hydrogen bonds between the base pairs in the Watson-Crick structure of DNA have a certain probability for tunneling, leading to discontinuous changes in the genetic code. Several computational studies have supported this hypothesis. ${ }^{10,12,41-43}$ The intramolecular PT mechanism has received a particular attention since nuclear quantum effects could inhibit the formation of certain tautomeric forms, which would avoid DNA 
damage. ${ }^{44}$ Yet, experimental evidences have indicated that tautomerism can provide a molecular explanation for the mutagenic properties. ${ }^{34}$ Moreover, the intermolecular tautomeric equilibrium in electronic excited states ${ }^{45}$ can favor specific tautomeric forms.

To clarify the spontaneous mutation mechanism proposed by Löwdin ${ }^{31}$, it is important to know that each nucleobase in DNA (or in RNA) can undergo a prototropic tautomerism, ${ }^{24}$ leading to keto-enol or amino-imino equilibria. As an example, the C tautomers is illustrated in the sketch below

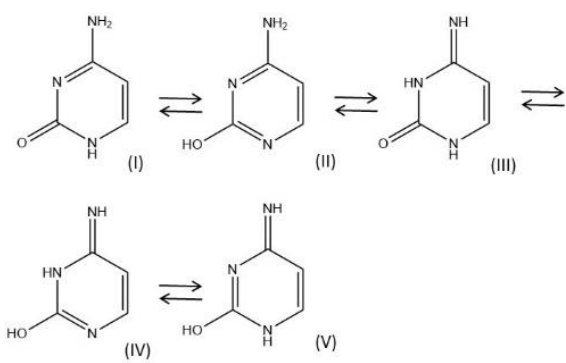

Some of these tautomers have recently been revealed in the gas phase by microwave spectroscopy combined with laser ablation techniques. ${ }^{46}$ Form I is commonly found in DNA, although forms III and V are possible to exist in proper conditions $^{24}$. The probability of occurring spontaneous mutation depends then on the possibility of DNA bases to form rare tautomeric forms. ${ }^{14}$ Hence, the role of modified bases present in DNA has an important functional meaning in gene expression. ${ }^{24}$

Interestingly, when a base pair is formed via hydrogen bonding in DNA strand, PT reactions may occur between the nucleobases, leading to their common tautomeric forms. This atomic phenomenon may be related to specific mutations during a DNA replication. ${ }^{31}$ In the following, we theoretically consider the SDPT in the GC pair:

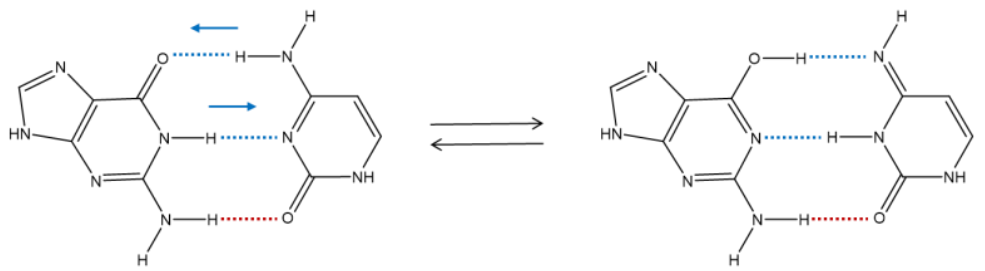


In this model, the base pairing is stabilized by three intermolecular hydrogen bonds and PT reactions can occur through the hydrogen bonds between the $\mathrm{G}$ and $\mathrm{C}$ molecules. However, during the SDPT, only two hydrogen atoms move from one side to the other of each hydrogen bond (dotted blue line in the sketch above), resulting in the most common $\mathrm{G}^{*} \mathrm{C}^{*}$ base pair, where $\mathrm{G}^{*}$ is the enol and $\mathrm{C}^{*}$ is the imino counterpart of $\mathrm{G}$ and $\mathrm{C}^{31}$, respectively.

Experimentally, Kwon and Zewail ${ }^{45}$ have investigated the dynamics of excitedstate DPT of 7-azaindole dimers (model DNA base pairs) in the condensed phase, by employing femtosecond fluorescence spectroscopy. Moreover, they have examined the effects of polarity and viscosity of the solvents involved in the process. In this sense, they have given evidence that the double proton transfer proceeds in a non-concerted way. Nevertheless, Takeuchi and Tahara ${ }^{47}$ have conducted similar experiments to those of Ref. ${ }^{45}$ in a nonpolar solvent and concluded that the DPT reaction is essentially a single-step process. Very recently, Bucher et al. ${ }^{48}$ have also detected a concerted decay of base pairs connected by hydrogen bonding in natural DNA. Most importantly, as can be understood from these experiments, the DPT tautomerism may be one of the underlying causes for point mutations during DNA replication.

Effects of a Gold Surface on the SDPT in GC. In order to gain insight on the impact of a gold surface in the PT of the GC pair, we have evaluated the SDPT mechanism: firstly in vacuum and finally adsorbed on the $\mathrm{Au}(111)$ surface. In Figure 5, we display the adiabatic potential energy profile of the double PT in the isolated GC pair. Our calculated transition state at $0.64 \mathrm{eV}(14.76 \mathrm{kcal} / \mathrm{mol})$ for the gas-phase $\mathrm{GC}$ $\mathrm{G}^{*} \mathrm{C}^{*}$ reaction is in line with reliable quantum-chemical estimates. ${ }^{14,49}$ Also, the energy difference between the $\mathrm{GC}$ and $\mathrm{G}^{*} \mathrm{C}^{*}$ forms at this level of calculation is around 0.36 $\mathrm{eV}(8.30 \mathrm{kcal} / \mathrm{mol})$, which confirms that $\mathrm{GC}$ is more stable than its $\mathrm{G}^{*} \mathrm{C}^{*}$ product, and it 
is in agreement with earlier quantum chemical procedures. ${ }^{14,50,51}$ In fact, the occurrence of the spontaneous point mutation via the tautomerism of the DNA bases remains a hot issue, ${ }^{49}$ mainly because the local influence upon the $\mathrm{GC} \leftrightarrow \mathrm{G}^{*} \mathrm{C}^{*}$ tautomerization is negligibly small. Furthermore, there is a lack of experimental data about the PT in isolated base pairs. Nonetheless, the effects of ligands, ${ }^{52}$ electron attachment ${ }^{53,54}$ or even the effect of an external field ${ }^{55}$ appear to be strong enough to modify the PT mechanism of DNA bases. In the following, we analyze the tautomerism in the GC base pair when it is in contact with an inert gold surface.

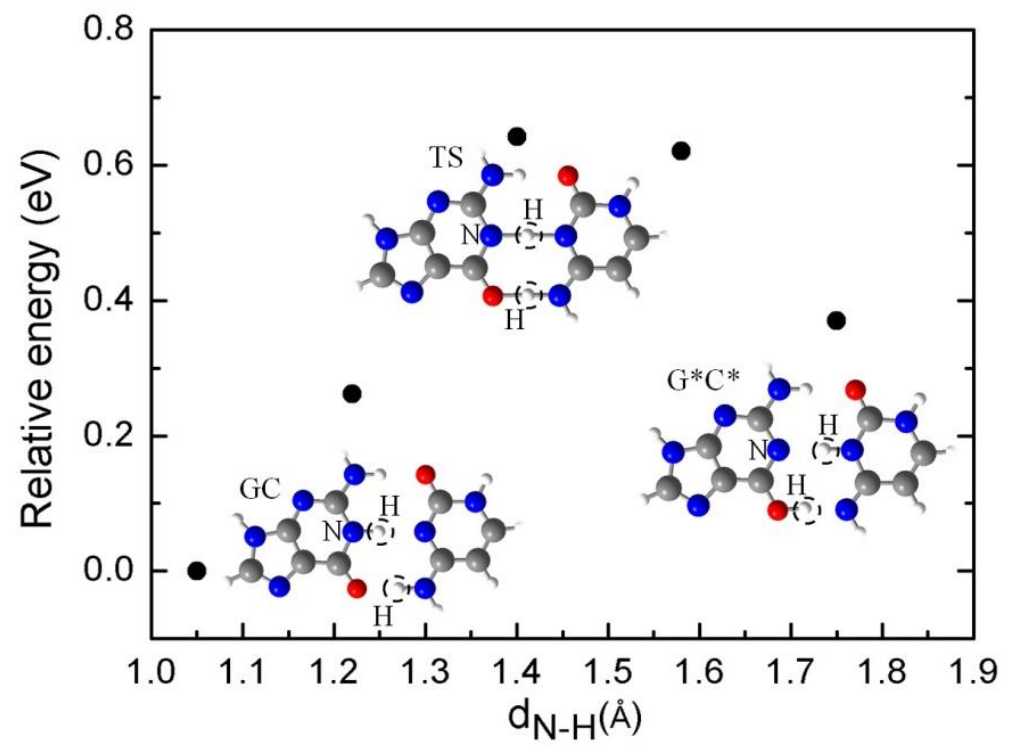

Figure 5. Gas-phase SDPT reaction profile from GC (a) up to $G^{*} C^{*}$ (b) calculated with the $v d W-D F^{30}$. The double proton-transfer starts to occur at the third point in the MEP, corresponding to an activation energy of $0.64 \mathrm{eV}$.

Now, considering the SDPT mechanism of GC at $\mathrm{Au}(111)$, we obtain a high reduction in its activation energy barrier of around 31\%, as displayed in Figure 6. In addition, the TS energy only becomes $0.04 \mathrm{eV}(0.92 \mathrm{kcal} / \mathrm{mol})$ higher than the $\mathrm{G}^{*} \mathrm{C}^{*}$ tautomer. The main effect of an $\mathrm{Au}(111)$ surface is to reduce the transition state energy to $0.44 \mathrm{eV}(10.15 \mathrm{kcal} / \mathrm{mol})$ for the $\mathrm{GC} \square \mathrm{G}^{*} \mathrm{C}^{*} / \mathrm{Au}(111)$ reaction, while the energy 
difference between the $\mathrm{GC}$ and $\mathrm{G}^{*} \mathrm{C}^{*}$ forms is slightly higher, around $0.40 \mathrm{eV}(9.22$ $\mathrm{kcal} / \mathrm{mol}$ ). This indicates that $\mathrm{GC}$ is still more stable than its $\mathrm{G}^{*} \mathrm{C}^{*}$ counterpart when adsorbed on a gold surface. However, the lowering of the activation energy of the GC tautomerism at $\mathrm{Au}(111)$ can trigger the occurrence of a point mutation during DNA replication, and appears to be in close connection with the mutagenic effects of gold nanoparticles. For example, this type of mutation induce aberrant phenotypes in Drosophila melanogaster and may be transmitted to the descendants. ${ }^{33}$ At this point, it is worth to mention that our calculations give evidence that a noble surface, such as $\mathrm{Au}(111)$, is enough to favor the PT in DNA bases. This is, however, a very different situation as compared to chemical modifications of nucleobases such as metal complexation or ionization of the GC pair. ${ }^{56}$

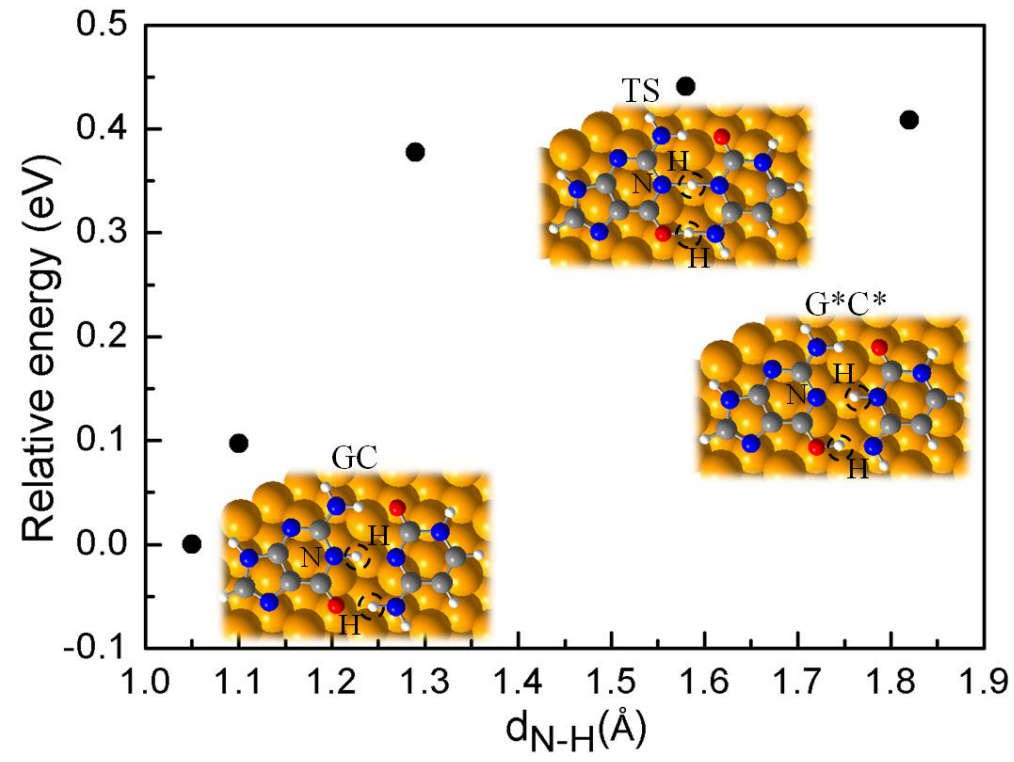

Figure 6. SDPT reaction profile from GC (a) up to $G^{*} C^{*}$ (b) at $\mathrm{Au}(111)$ (hcp site) calculated with the vdW-DF ${ }^{30}$. The double proton transfer starts to occur at the fourth point in the MEP, corresponding to an activation energy of $0.44 \mathrm{eV}$.

\section{CONCLUSIONS}


We have presented a comprehensive study on the SDPT in the GC base pair adsorbed on an $\mathrm{Au}(111)$ surface, based on first-principles calculations. Our results indicate significant differences in the electronic structure, energetics and kinetics of the tautomerism of the GC pair, when in contact with an inert gold surface, which can modify the SDPT mechanism. We have determined a reduction of about $31 \%$ in the activation energy barrier of the PT in $\mathrm{GC} / \mathrm{Au}(111)$. Furthermore, the energy differences between the TS and $\mathrm{G}^{*} \mathrm{C}^{*}$ tautomer are significantly decreased, favoring the enol and imino forms. Although the occurrence of spontaneous point mutation due to the tautomerism of DNA base pair continues to be a controversial issue in biochemistry, mainly because of the lack of experimental data for isolated base pairs, our findings are in line with a recent experimental study on the mutagenic effects of gold nanoparticles in the phenotype of Drosophila melanogaster. ${ }^{33}$ Most importantly, our computational study is closely related with recent experiments conducted by $\mathrm{Li}$ et al., ${ }^{34}$ which correlates mutagenicity and tautomerism in the anti-HIV nucleoside KP1212. Finally, due to the growing applications of gold nanoparticle in the human organism, ${ }^{57}$ including via cosmetics, ${ }^{58}$ we stress that the role of these supposed inert nanomaterials deserve further investigation before to be widespread as alternative medical applications.

\section{ASSOCIATED CONTENT}

Supporting Information Available: Calculated average intermolecular distances, binding energies, Bader charges, and work functions for the GC pair adsorbed on distinct sites of $\mathrm{Au}(111)$. Calculations without vdw corrections are reported for the electrostatic potential, PDOS, and kinetics of the SDPT of GC/Au(111). This material is available free of charge via the Internet at http://pubs.acs.org 


\section{AUTHOR INFORMATION}

\section{Corresponding Authors}

*E-mail: rivelino@ufba.br. Tel: +55 71 3283-6640. Fax: +55 713283 66-06

*E-mail: caio@ufba.br. Tel: +55 71 3283-6640

\section{Notes}

The authors declare no competing financial interest.

\section{ACKNOWLEDGMENTS}

The authors gratefully acknowledge financial support by the Swedish Research Council (VR) through Swedish Research Links project 348-2014-4249. G.K.G. gratefully acknowledges support by the Linköping Linnaeus Initiative for Novel Functionalized Materials (LiLi-NFM, VR) as well as by the Swedish Foundation for Strategic Research (SSF) Synergy Grant \#RMA11-0029 on Functional Carbides and Advanced Surface Engineering (FUNCASE). R.R., C.M.C.deC., and F.deB.M. acknowledge Conselho Nacional de Desenvolvimento Científico e Tecnológico (CNPq) and Fundação de Amparo à Pesquisa do Estado da Bahia (FAPESB) for the financial support. R.R.Q.F. acknowledges the financial support by Coordenação de Aperfeiçoamento de Pessoal de Nível Superior (CAPES). 


\section{REFERENCES}

1. Sowerby, S. J.; Heckl, W. M. The Role of Self Assembled Purine and Pyrimidine Bases in the Emergence of Life. Origins Life Evol. Biosphere 1998, 28, 283-310.

2. Sowerby, S. J.; Cohn, C. A.; Heckl, W. M.; Holm, N. G. Differential Adsorption of Nucleic Acid Bases: Relevance to the Origin of Life. Proc. Natl. Acad. Sci. USA 2001, $98,820-822$.

3. Ohtomo, Y.; Kakegawa, T.; Ishida, A.; Nagase, T.; Rosing, M. T. Evidence for Biogenic Graphite in Early Archaean Isua Metasedimentary Rocks. Nature Geosci. 2014, 7, 25-28.

4. Somorjai, G. A. Introduction to Surface Chemistry and Catalysis. John Wiley \& Sons: New York, 1994.

5. Aradhya, S. V.; Frei, M.; Hybertsen, M. S.; Venkataraman, L. Van der Waals Interactions at Metal/Organic Interfaces at the Single-Molecule Level. Nature Mat. 2012, 11, 872-876.

6. Shao, K.; Singha, S.; Clemente-Casares, X.; Tsai, S.; Yang, Y.; Santamaria, P. Nanoparticle-Based Immunotherapy for Cancer. ACS Nano 2015, 9, 16-30.

7. Sykes, E. A.; Chen, J.; Zheng, G.; Chan, W. C. W. Investigating the Impact of Nanoparticle Size on Active and Passive Tumor Targeting Efficiency. ACS Nano 2014, 8, 5696-5706.

8. Chithrani, B. D.; Chan, W. C. W. Elucidating the Mechanism of Cellular Uptake and Removal of Protein-Coated Gold Nanoparticles of Different Sizes and Shapes. Nano Lett. 2007. 7, 1542-1550. 
9. Sun, W.; Ferretti, A.; Varsano, D.; Brancolini, G.; Corni, S.; Di Felice, R. Charge Transfer Rates at a Bio-Inorganic Interface. J. Phys. Chem. C 2014, 118, 18820-18828.

10. Kosenkov, D.; Kholod, Y.; Gorb, L.; Shishkin, O.; Hovorun, D. M.; Mons, M.; Leszczynski, J. Ab Initio Kinetic Simulation of Gas-Phase Experiments: Tautomerization of Cytosine and Guanine. J. Phys. Chem. B 2009, 113, 6140-6150.

11. Zelený, T.;Ruckenbauer, M.; Aquino, A. J.; Müller, T.; Lankaš, F.; Dršata, T.; Hase, W. L; Nachtigallova, D.; Lischka, H. Strikingly Different Effects of Hydrogen Bonding on the Photodynamics of Individual Nucleobases in DNA: Comparison of Guanine and Cytosine. J. Am. Chem. Soc. 2012, 134, 13662-13669.

12. Colominas, C.; Luque, F. J.; Orozco, M. Tautomerism and Protonation of Guanine and Cytosine. Implications in the Formation of Hydrogen-Bonded Complexes. J. Am. Chem. Soc. 1996, 118, 6811-6821.

13. Villani, G. Coupling between Hydrogen Atoms Transfer and Stacking Interaction in Adenine-Thymine/Guanine-Cytosine Complexes: A Theoretical Study. J. Phys. Chem. B 2014, 118, 5439-5452.

14. Gorb, L.; Podolyan, Y.; Dziekonski, P.; Sokalski, W. A.; Leszczynski, J. DoubleProton Transfer in Adenine-Thymine and Guanine-Cytosine Base Pairs. A PostHartree-Fock ab Initio Study. J. Am. Chem. Soc. 2004, 126, 10119-10129.

15. Maleki, A.; Alavi, S.; Najafi, B. Molecular Dynamics Simulation Study of Adsorption and Patterning of DNA Bases on the Au(111) Surface. J. Phys. Chem. C 2011, 115, 22484-22494. 
16. Otero, R.; Schöck, M.; Molina, L. M.; Lægsgaard, E.; Stensgaard, I.; Hammer, B.; Besenbacher, F. Guanine Quartet Networks Stabilized by Cooperative Hydrogen Bonds. Angew. Chem. Int. Ed. 2005, 44, 2270-2275.

17. Koch, N.; Ueno, N.; Wee, A. T. S.; Eds. The Molecule-Metal Interface. WileyVCH: Weinheim, 2013.

18. Karan, S.; Wang, Y.; Robles, R.; Lorente, N.; Berndt, R. Surface-Supported Supramolecular Pentamers. J. Am. Chem. Soc. 2013, 135, 14004-14007.

19. Otero, R.; Lukas, M.; Kelly, R. E. A.; Xu, W.; Lægsgaard, E.; Stensgaard, I.; Kantorovich, L. N.; Besenbacher, F. Elementary Structural Motifs in a Random Network of Cytosine Adsorbed on a Gold(111) Surface. Science 2008, 319, 312-315.

20. Kelly, R. E. A.; Xu, W.; Lukas, M.; Otero, R.; Mura, M.; Lee, Y.-J.; Lægsgaard, E.; Stensgaard, I.; Kantorovich, L. N.; Besenbacher, F. An Investigation into the Interactions between Self-Assembled Adenine Molecules and a Au(111) Surface. Small 2008, 4, 1494-1500.

21. Xu, W.; Wang, J.; Jacobsen, M. F.; Mura, M.; Yu, M.; Kelly, R. E. A.; Meng, Q.; Lægsgaard, E.; Stensgaard, I.; Linderoth, T. R; et al. Supramolecular Porous Network Formed by Molecular Recognition between Chemically Modified Nucleobases Guanine and Cytosine. Angew. Chem. Int. Ed. 2010, 49, 9373-9377.

22. Smerieri, M.; Vattuone, L.; Costa, D.; Tielens, F.; Savio, L. Self-Assembly of (S)Glutamic Acid on Ag(100): A Combined LT-STM and Ab Initio Investigation. Langmuir 2010, 26, 7208-7215. 
23. Wang, Z.; Sun, N.; He, Y.; Liu, Y.; Li, J. DNA Assembled Gold Nanoparticles Polymeric Network Blocks Modular Highly Sensitive Electrochemical Biosensors for Protein Kinase Activity Analysis and Inhibition. Anal. Chem. 2014, 86, 6153-6159.

24. Griffin, B. E.; Structure of DNA and its Relationship to Carcinogenesis. In Introduction to the Cellular and Molecular Biology of Cancer; Franks, L. M.; Teich, N. M.; Eds.; Oxford University Press: Oxford, 1997; 3rd edn, pp 92-105.

25. Kong, H.; Sun, Q.; Wang, L.; Tan, Q.; Zhang, C.; Sheng, K.; Xu, W. Atomic-Scale Investigation on the Facilitation and Inhibition of Guanine Tautomerization at $\mathrm{Au}(111)$ Surface. ACS Nano 2014, 8, 1804-1808.

26. Kresse, G.; Hafner, J. Ab Initio Molecular Dynamics for Liquid Metals. Phys. Rev. B 1993, 47, 558-561.

27. Kresse, G.; Furthmüller, J. Efficient Iterative Schemes for Ab Initio Total-Energy Calculations Using a Plane-Wave Basis Set. Phys. Rev. B 1996, 54, 11169-11186.

28. Kresse, G.; Joubert, D. From Ultrasoft Pseudopotentials to the Projector Augmented-Wave Method. Phys. Rev. B 1999, 59, 1758-1775.

29. Perdew, J. P.; Burke, K.; Ernzerhof, M. Generalized Gradient Approximation Made Simple. Phys. Rev. Lett. 1996, 77, 3865.

30. Klimeš, J.; Bowler, D. R.; Michaelides, A. Van der Waals Density Functionals Applied to Solids. Phys. Rev. B 2011, 83, 195131.

31. Löwdin, P.-O. Proton Tunneling in DNA and Its Biological Implications. Rev. Mod. Phys. 1963, 35, 724-732.

32. Tomasetti, C.; Vogelstein, B. Variation in Cancer Risk among Tissues Can Be Explained by the Number of Stem Cell Divisions. Science 2015, 347, 78-81. 
33. Vecchio, G.; Galeone, A.; Brunetti, V.; Maiorano, G.; Rizzello, L.; Sabella, S.; Cingolani, R.; Pompa, P. P. Mutagenic Effects of Gold Nanoparticles Induce Aberrant Phenotypes in Drosophila melanogaster. Nanomed. Nanotech. Biol. Med. 2012, 8, 1-7.

34. Deyu Li, D.; Fedeles, B. I.; Singh, V.; Peng, C. S.; Silvestre, K. J.; Simi, A. K.; Simpson, J. H.; Tokmakoff, A.; Essigmann, J. M. Tautomerism Provides a Molecular Explanation for the Mutagenic Properties of the Anti-HIV Nucleoside 5-aza-5,6dihydro-2'-deoxycytidine. Proc. Natl. Acad. Sci. USA 2014, 111, E3252-E3259.

35. Jónsson, H.; Mills, G.; Jacobsen, K. W. In Classical and Quantum Dynamics in Condensed Phase Simulations; Berne, B. J., Ciccotti, G., Coker, D. F., Eds.; World Scientific: Singapore, 1998.

36. González-García, N.; Pu, J.; González-Lafont, A.; Lluch, J. M.; Truhlar. D. G. Searching for Saddle Points by Using the Nudged Elastic Band Method: An Implementation for Gas-Phase Systems. J. Chem. Theory Comput. 2006, 2, 895-904.

37. Henkelman, G.; Uberuaga, B. P.; Jónsson, H. A Climbing Image Nudged Elastic Band Method for Finding Saddle Points and Minimum Energy Paths. J. Chem. Phys. 2000, 113, 9901.

38. Livshits, A. I.; Kantorovich, L. Guanine Assemblies on the Au(111) Surface: A Theoretical Study. J. Phys. Chem. C 2013, 117, 5684-5692.

39. Tang, W.; Sanville, E.; G Henkelman. A Grid-Based Bader Analysis Algorithm without Lattice Bias. J. Phys.: Condens. Matter 2009, $21,084204$.

40. Sony, P.; Puschnig, P.; Nabok, D.; Ambrosch-Draxl, C. Importance of van der Waals Interaction for Organic Molecule-Metal Junctions: Adsorption of Thiophene on $\mathrm{Cu}(110)$ as a Prototype. Phys. Rev. Lett. 2007, 99, 176401. 
41. Jacquemin, D.; Zúñiga, J.; Requena, A.; Céron-Carrasco, J. P. Assessing the Importance of Proton Transfer Reactions in DNA. Acc. Chem. Res. 2014, 47, $2467-2474$.

42. Xiao, S.; Wang, L.; Liu, Y.; Lin, X.; Liang, H. Theoretical Investigation of the Proton Transfer Mechanism in Gguanine-Cytosine and Adenine-Thymine Base Pairs. $J$. Chem. Phys. 2012, 137, 195101.

43. V. Sauri, J. P. Gobbo, J. J. Serrano-Pérez, M. Lundberg, P. B. Coto, L. SerranoAndrés, A. C. Borin, R. Lindh, M. Merchán, D. Roca-Sanjuán. Proton/Hydrogen Transfer Mechanisms in the Guanine-Cytosine Base Pair: Photostability and Tautomerism. J. Chem. Theory Comput. 2013, 9, 481-496.

44. Pérez, A.; Tuckerman, M. E.; Hjalmarson, H. P.; von Lilienfeld, O. A. Enol Tautomers of Watson-Crick Base Pair Models Are Metastable Because of Nuclear Quantum Effects. J. Am. Chem. Soc. 2010, 132, 11510-11515.

45. Kwon, O.-H.; Zewail, A. H. Double Proton Transfer Dynamics of Model DNA Base Pairs in the Condensed Phase. Proc. Natl. Acad. Sci. USA 2007, 104, 8703-8708.

46. Alonso, J. L.; Vaquero, V.; Peña, I.; López, J. C.; Mata, S.; Caminati, W. All Five Forms of Cytosine Revealed in the Gas Phase. Angew. Chem. Int. Ed. 2013, 52, 1-5.

47. Takeuchi, S.; Tahara, T. The Answer to Concerted versus Step-Wise Controversy for the Double Proton Transfer Mechanism of 7-Azaindole Dimer in Solution. Proc. Natl. Acad. Sci. USA 2007, 104, 5285-5290.

48. Bucher, D. B.; Schlueter, A.; Carell, T.; Zinth, W. Watson-Crick Base Pairing Controls Excited-State Decay in Natural DNA. Angew. Chem. Int. Ed. 2014, 53, 11366 $-11369$. 
49. Brovarets, O. O.; Hovorun, D. M. Why the Tautomerization of the G.C WatsonCrick Base Pair via the DPT Does Not Cause Point Mutations during DNA Replication? QM and QTAIM Comprehensive Analysis. J. Biomol. Struct. Dyn. 2014, 32, 14741499.

50. Florian, J.; Hrouda, V.; Hobza, P. Proton Transfer in the Adenine-Thymine Base Pair. J. Am. Chem. Soc. 1994, 116, 1457-1460.

51. Florián, J; Leszczyński, J. Spontaneous DNA Mutations Induced by Proton Transfer in the Guanine-Cytosine Base Pairs: An Energetic Perspective. J. Am. Chem. Soc. 1996, $118,3010-3017$.

52. Fujikawa, Y.; Kawanishi, M.; Kuraoka, I.; Yagi, T. Frequencies of Mutagenic Translesion DNA Synthesis over Cisplatin-Guanine Intra-Strand Crosslinks in lacZ Plasmids Propagated in Human Cells. Mutat. Res. Genet. Toxicol. Environ. Mutagen. $\mathbf{2 0 1 4}, 770,23-28$.

53. Chen, H.-Y.; Kao, C.-L.; Hsu, S. C. N. Proton Transfer in Guanine-Cytosine Radical Anion Embedded in B-Form DNA. J. Am. Chem. Soc. 2009, 131, 1593015938

54. Bera, P. P.; Schaefer III, H. F. $(\mathrm{G}-\mathrm{H}) \bullet-\mathrm{C}$ and $\mathrm{G}-(\mathrm{C}-\mathrm{H}) \bullet$ Radicals Derived from the Guanine. Cytosine Base Pair Cause DNA Subunit Lesions. Proc. Natl. Acad. Sci. USA 2005, 102, 6698-6703.

55. Cerón-Carrasco, J. P.; Jacquemin, D. Electric Field Induced DNA Damage: An Open Door for Selective Mutations. Chem. Commun. 2013, 49, 7578-7580. 
56. Yamamura, M.; Ichino, T.; Yoshioka, Y. A B3LYP Study on Repair of Guanyl and 8-Oxoguanyl Radical by Simultaneous Proton- and Electron-Transfer Reaction. Bull. Chem. Soc. Jpn. 2011, 84, 181-190.

57. Mironava, T.; Hadjiargyrou, M.; Simon, M.; Rafailovich, M. H. Gold Nanoparticles Cellular Toxicity and Recovery: Adipose Derived Stromal Cells. Nanotoxicology 2014, 8, 189-201.

58. Kim, J.-H.; Hong, C.-O.; Koo, Y.-C.; Choi, H.-D.; Lee, K.-W. Anti-Glycation Effect of Gold Nanoparticles on Collagen. Biol. Pharm. Bull. 2012, 35, 260-264. 
TABLE OF CONTENTS GRAPHIC

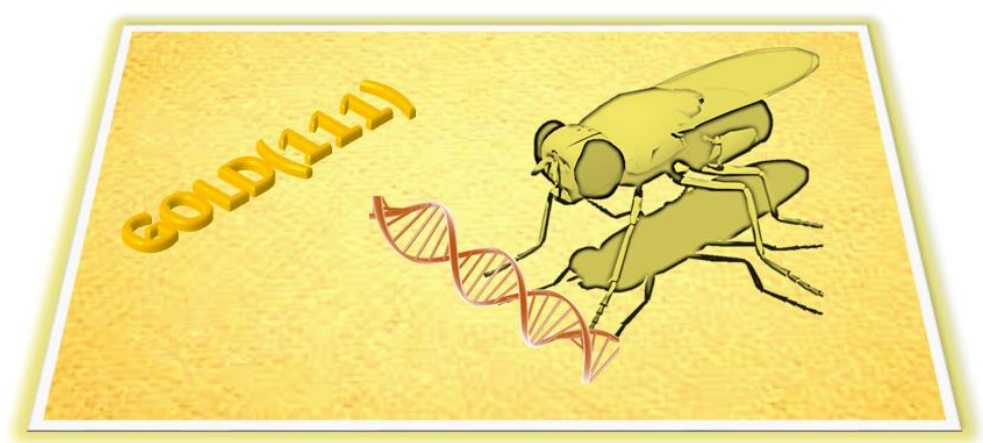

